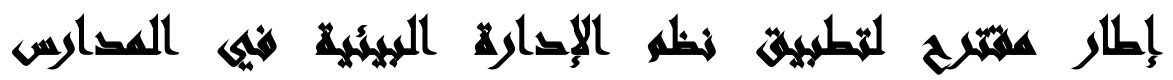

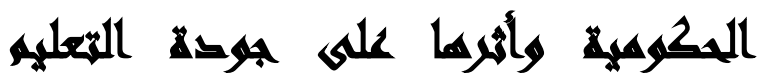

[lv]

جمال سعد خطاب(')- ريهام رفعت محمد(ץ)- سحر نور الدين محمد صالح

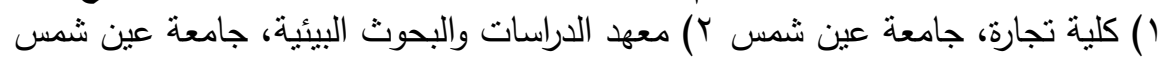

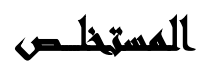

تسعى نظم الإدارة البيئية المدرسية إلى تكوين الإنسان الذي ينهع بالمهارات والاتجاهات

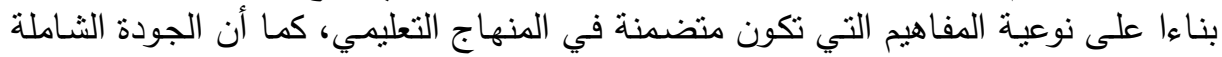

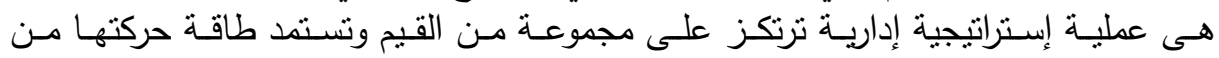

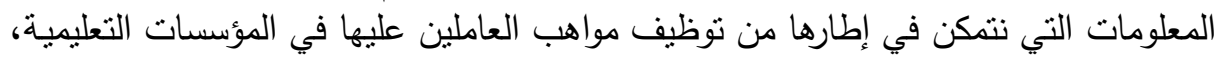

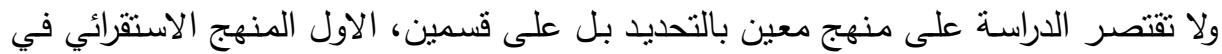

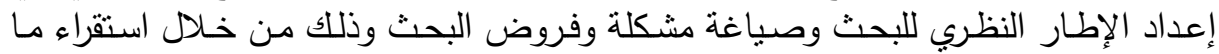

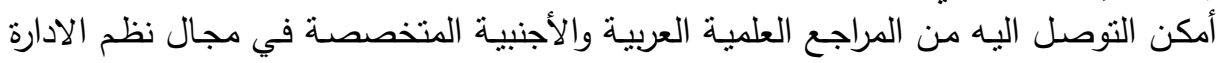

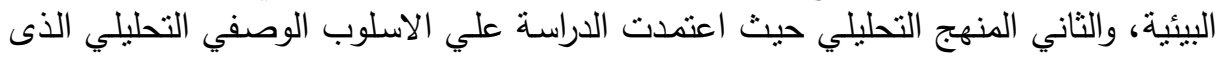

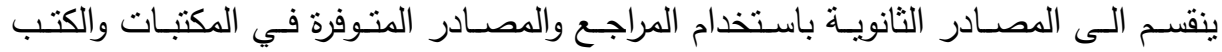

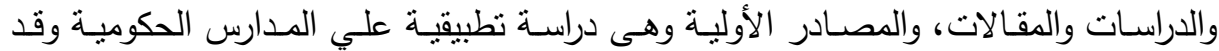

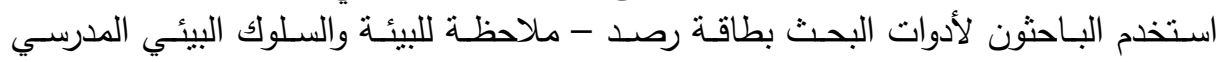

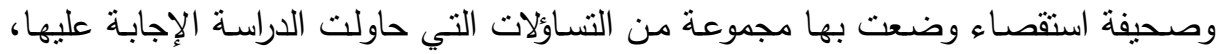

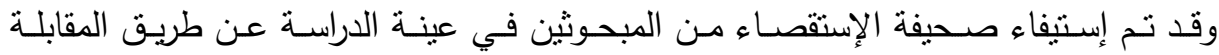

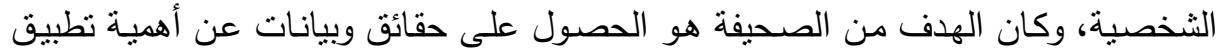

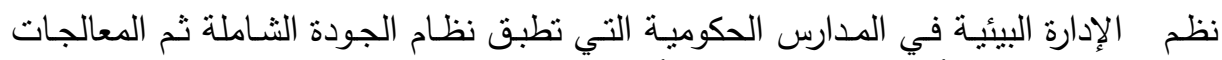

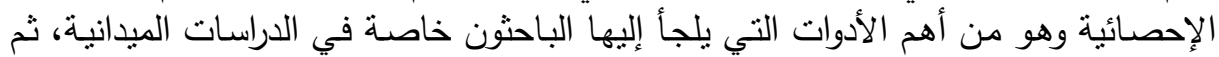

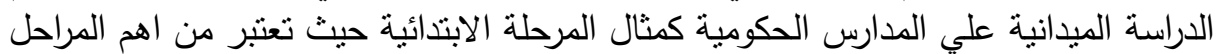

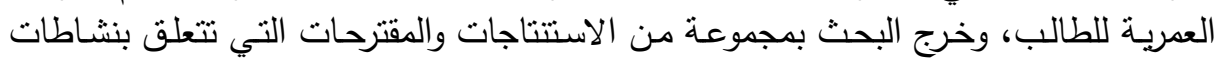

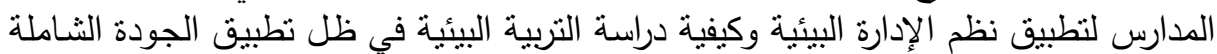

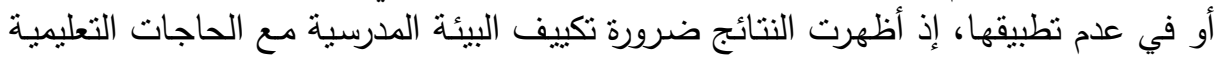

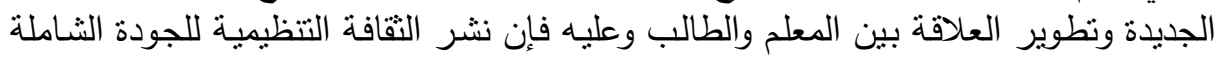

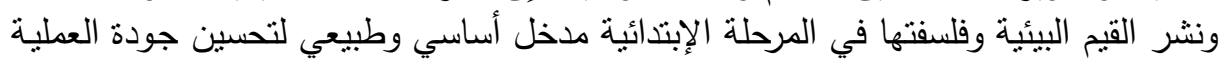




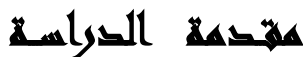

تشهد التربية اليوم في عصر المعرفة نطورات جوهرية نتتاول مكونات النظام التربوي برمته بالتعديل والتطوير منها: التزكيز على المتعلم بصورة أساسية والتتويع في عمليات التعلم على حساب التعليم، والانتقال من دور المعلم الملقن والمحفظ إلى دور الميسر والمدرب، والتركيز على التعلم الذاتي في مجال تطبيق التربية البيئية في المدارس خاصة مدارس التعليم الأساسي، والتأكيد على النشاط والممارسة على أنها مداخل واستراتيجيات تربوية فاعلة وانطلاقا من ذلك فإن دراسة منظومة التعليم البيئي لمختلف فئاته إما تساعد علي فشل العملية التعليمية أو نجاحها من خلال ما هو مناح من تعليم وقرارات ولوائح وإمكانيات، الأمر الذي ينعكس علي سلوك الطلبة والطالبات في شتي مجالات الحياة. فالإدارة البيئية السليمة هى تلاك التي تتطوي على التخطيط البيئي السليم والذي يتماشى

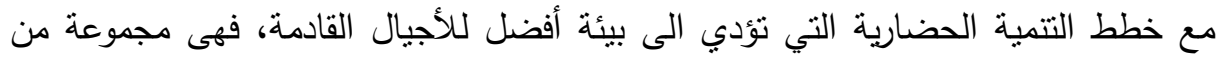

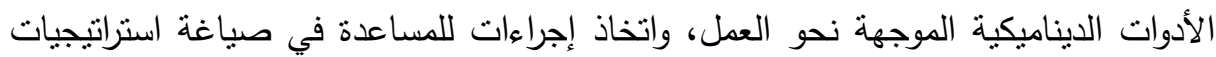
لحماية البيئة وتعزيزها وصيانتها ومن ثم تتفيذ الاستراتيجيا ومراقبتها. كما يعد التعليم من العوامل الاساسية التي ينتقي منها الأفراد كل ثقافتهم سواء

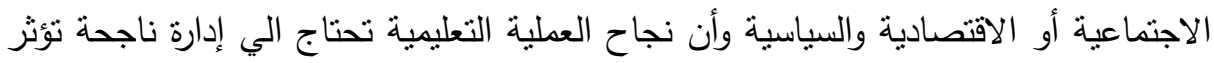
بشكل ايجابي في المجتمعات المختلفة، قد عُنيت الدراسات الحديثة في مجال التغييرات التئهات في نظام التعليم بتقييم المحاولات التي جرت في بعض البلاد المتقدمة تحت شعار إصلاح التعليم أو تطوير التعليم .وقررت بعض الدراسات قصور هذه المحاولات في تحقيق الأهداف المنشودة

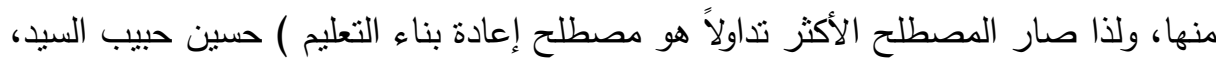

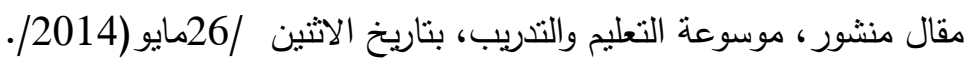

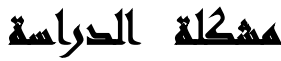

يمكن صباغة المشكلة إلي أن بعض المدارس الحكومية قد يكون هناك تطبيق لنظم الإدارة البيئية ولكن ليس بالثكل المأمول، وكذلك هناك مدارس أخري لا تطبق نظم الإدارة 
البيئية مطلقاً، أو وجود مدارس ليس لها دراية بوجود نظم إدارة بيئية من الاساس، وبالتالي

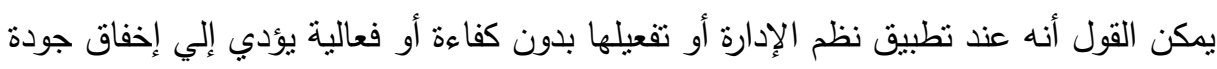

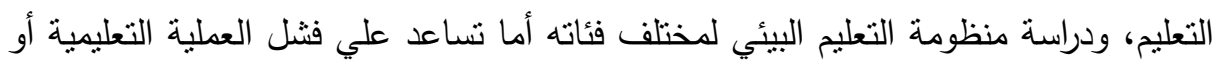

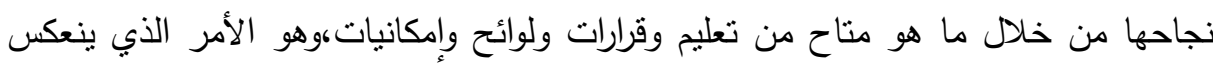

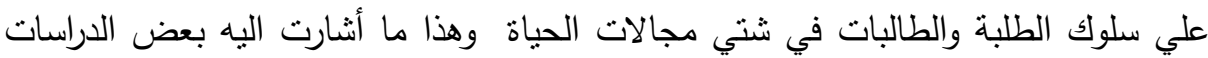

السابقة.

1-دراسة للأستاذ خالا عبد السميع قاسم- بعنوان: الجودة والاعتماد التريوي إصدار2010: وأشار الى ان الادارة البيئية هي مدخل لتحسين منظومة العملية التعليمية بوجه عام بما تتضمنه من معلم ومتعلم وإدارة مدرسية ومبني مدرسي وبيئة تعليمية ومناخ مدرسي، وما يتطلبه ذلك من دعم وتحسين العمليات التعليمية وتكنولوجيا التعليم مما يسهم في تحسين مخرجات التعليم.

r-دراسة: إعداد .د .حسن ابو بكر - إصدار نوفمبر 2012عنوانه: التهج الحقوقي في التنمية وتطبيقاته في مجالات البيئة: من اهم أهداف الدراسة:مراقبة

حقوق الطلبة في بيئة خالية من الاضرار داخل المدرسة. - - رفع وعي عدد من الطلبة وأولياء الأمور والمعلمين بالأضرار البيئية - - إلزام مديري المدارس والمؤسسات التعليمبة بتطبيق القانون - تفعيل الادارة البيئية المدرسية يؤدي إلي النأثير بإيجابية علي جودة التعليم وعلى أنز ونهج الدراسات السابقة هدفت الدراسة الى توضيح دور نظم الإدارة البيئية داخل المدارس فى صورة لبعض الاسئلة .

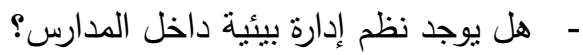
- - - هل تطبق تلك النظم؟ - ما - ما الطرق لتفعيل تطبيق نظم الإدارة البيئية؟

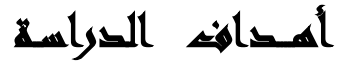


الهدف الرئيسي للاراسة هو التعرف على نظم الإدارة البيئية والأسباب التي ادت إليها،

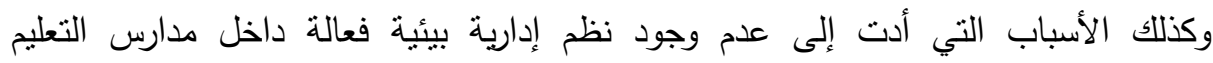
الاساسي التي تطبق الجودة الثشاملة في التعليم والتي لم تطبق الجودة في التعليم، وتوضيح ذلك عملياً من خلال الدراسة الميدانية.

\section{أهمية الصواسـا}

الأهمية النظرية: يرجع إلي فاعلية نظم الإدارة البيئية كنظام تعليمي يؤدي بدورة إلي جودة التعليم ودعم كافة المتعلمين والقائمين علي العملية التعليمية، لأنه في الدرجة الأولي يهتم بالبيئة المحيطة بكل من الطالب والمدرس. الأهمية العملية: أن يكون للمدرسة مبادئها التي تلتزم بتطبيقها بهدف تحسين الاداء بشكل مستمر ومنه إلي تحسين التعليم.

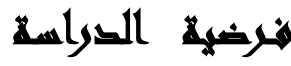

توجد علاقة ارتباط موجبة بين تطبيق نظم الإدارة البيئية فى المدارس الحكومية وتحسين

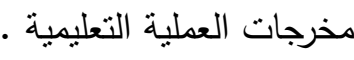
الفرض الأول: يوجد علاقة ارتباط موجبة بين تطبيق نظم الإدارة البيئية في المدارس الحكومية وتحسين مخرجات العطية التعليمية. الفرض الثاني: يؤدي وجود معوقات لتطبيق نظم الإدارة البيئية إلى ضعف مخرجات العملية التعليمية. 


\section{الإجباءاهي المنهجية للسواسمة}

منهج الاراسة: ينقسم إلي قسمين:

القسم الأول: المنهج الاستقرائي: حيث اعتمد البحث علي المنهج الاستقرائي في إعداد

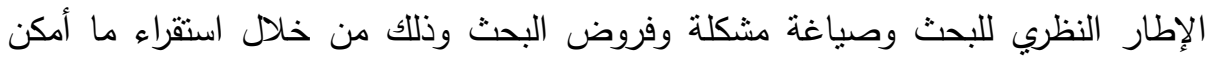

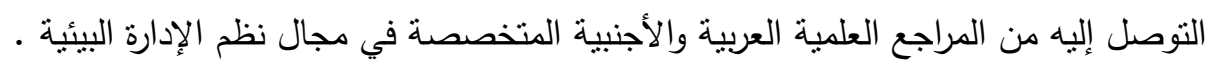
القسم الثاني المنهج التحليلي: اعتمدت الدراسة علي الأسلوب الوصفي التحليلي من النوع التيه المسحي نظرا لملاعمة هذا المنهج لمثال هذه الدراسات، حيث اعتمدت علي المصادر الآتية :

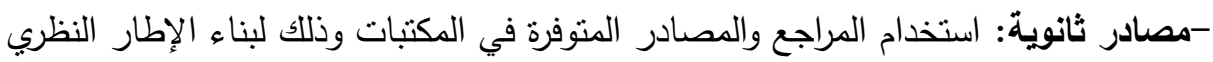
لهذه الدراسة ومنها الكتب والدراسات والمقالات. -مصادر أولية: دراسة ميدانية علي مدارس التعليم الاساسي التي تطبق الجودة في التعليم

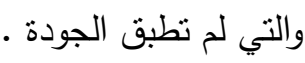

\section{أسوايت الفراسمة}

وقد استخدم الباحثون في الدراسة الراهنة مجموعة من الأدوات أهمها:

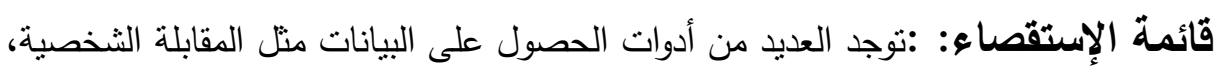
والمكالمات التليفونية، والبريد الإلكتروني، وقوائم الاستقصاء ...إلخ، وقد اعنمد الباحثون في لئي

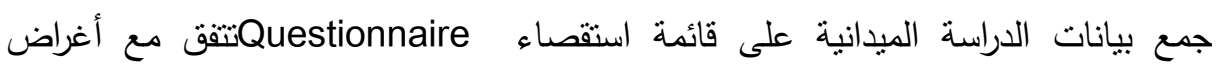

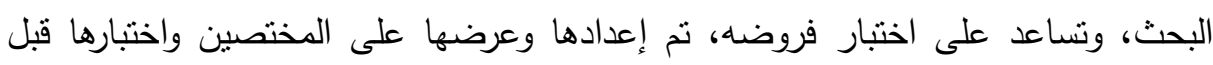

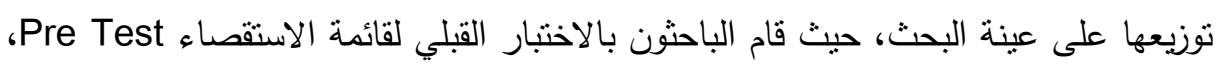

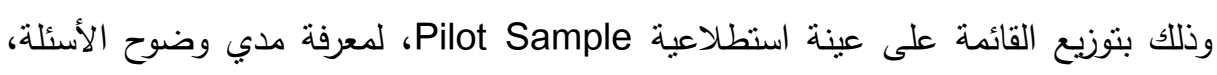
وملاحظات المستجيبين، وقد استخدم الباحثون أسلوب المقابلة الثخصية في هذه المرحلة، وبعد التأكد من ملاءمة قائمة الاستقصاء لأهداف وفروض البحث قام الباحثون بصياغتها في

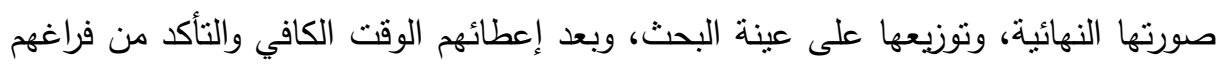
من استكمال هذه القوائم تم جمع هذه القوائم والقيام بتحليلها، ولضمان عيدان حيادية الاستجابات أكد

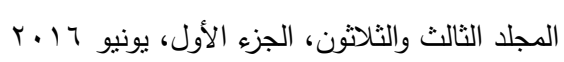


الباحثون على غلاف القائمة أن جميع الإجابات ستكون سرية ويقتصر استخدامها على

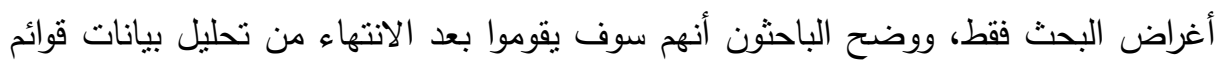

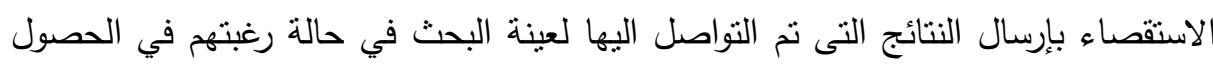
على هذه النتائج. وكانت استمارة الاستقصاء عبارة عن ثناث محاور رئيسية وهم:

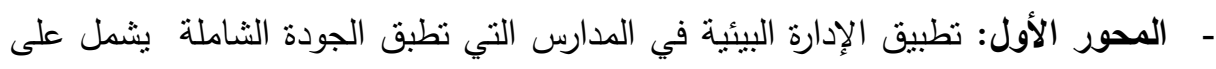
19 سؤال - - المحور الثانى: معوقات نطبيق الإدارة البيئية ونظام الجودة في المدارس الحكومية وسبل

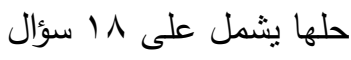
- - المحور الثالث: تأثثر تطبيق نظم الإدارة البيئية على جودة التعليم ويشمل على ع اسئلة فقط - وتم توزيع الاستمارة على المختصون بالعملية التعليمية ببعض المدارس الحكومية بالمرحلة الابتدائية بمحافظة القاهرة. - تم الحصول على عينة من ب † ب فرداً من مجتمع الدراسة شملت الددرسين والوكلاء والمديرين والأخصائيين. كاتت نتيجة استخدام إستمارة الاستقصاء كما يلى: والى اختبار الفرض الأول: يوجد علاقة ارتباط موجبة بين تطبيق نظم الإدارة البيئية في المداس الاستئي الحكومية، وتحسين مخرجات العملية التعليمية. وقد تم اختبار هذا الفرض من خلال دراسة علاقة الارتباط والانحدار بين المتغير التابع "جودة التعليم "والمتغيرات المستقلة "تطبيق الإدارة البيئية "وجاءت التهن النتائج كما يلي: علاقات الارتباط بين المتغير التابع والمتغيرات المستقلة: بدرسة علاقة الارتباط بين المتغير وادير

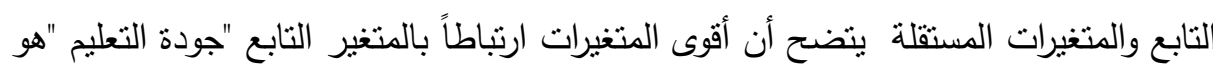

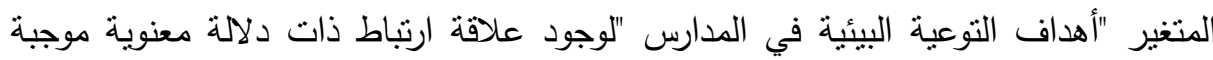

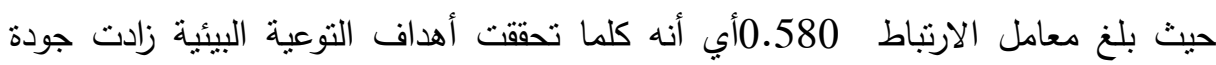


التعليم، يلي ذلك المتغير "التقنيات التربوية "حيث وجدت علاقة ارتباط ذات دلالة معنوية

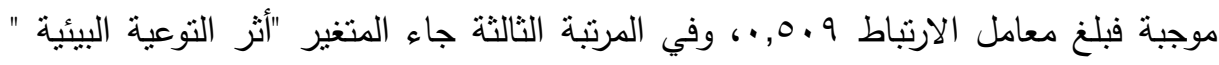

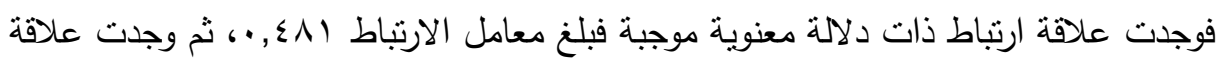

ارتباط ذات دلالة معنوية موجبة بين "جودة التعليم "والمتغير "تطبيق الإدارة البيئية " نخلص من ذللك إلى وجود علاقة ارتباط ذات دلالة معنوية موجبة بين المتغير التابع

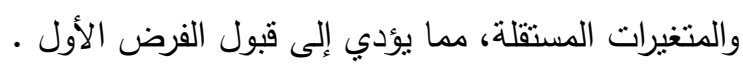

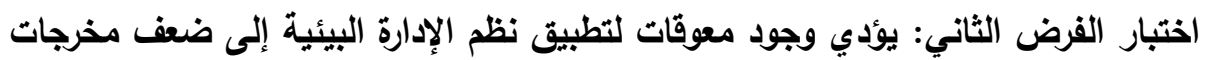
العملية التعليمية. وقد تم اختبار هذا الفرض من خلال دراسة علاقة الارتباط والانحدار بين المتغير التابع

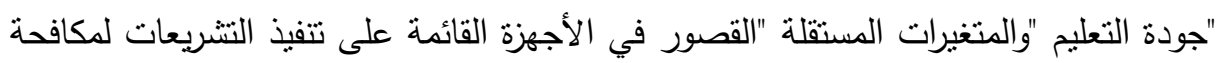

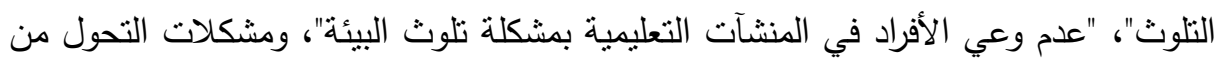

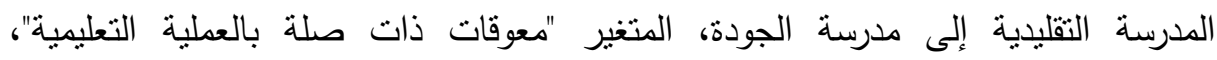

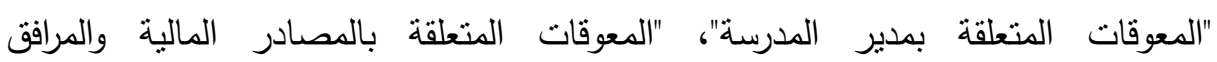
والتجهيزات"، "المعوقات المتعلقة بالعلاقة بين المدرسة ووزارة التربية والتعليم"، وجاءت النتائج كما يلي:

أكثر المعوقات تأثيراً على جودة التعليم هو المتغير "المعوقات المتعلقة بالمصادر المالية والمرافق والتجهيزات "حيث وجدت علاقة ذات دلالة معنوية سالبة حيث بلغ معامل الارتباط -

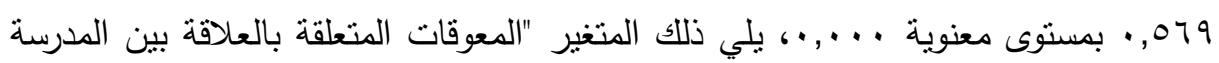

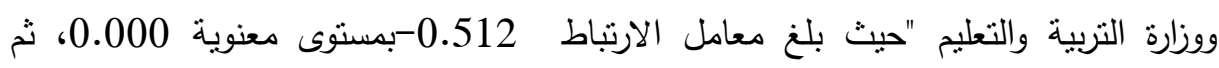
المتغير " المعوقات المتعلقة بمدير المدرسة "والمتغير "معوقات ذات صلة بالعملية التعليمية "

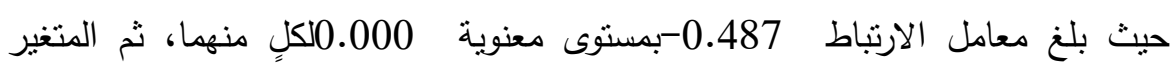

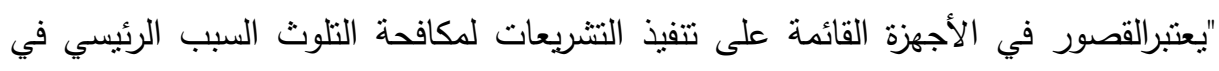

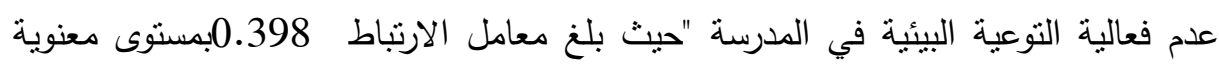


Xيتسبب عدم وعى الأفراد في المنشآت التعليمية بمشكلة تلوث

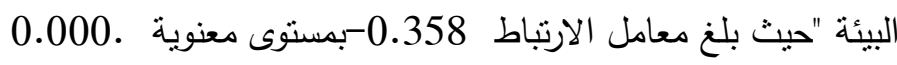

نخلص من ذللك إلى وجود علاقة ارتباط ذات دلالة معنوية سالبة بين المتغير التابع

والمتغيرات المستقلة، مما يؤدي إلى قبول الفرض الثناني .

\section{هجالائش التولسم}

المجال المكاني: داخل بعض المدارس الحكومية للتعليم الأساسي التي تطبق نظام الجودة الثناملة في التعليم بمدارس محافظة القاهرة والتي تطبق نظم الإدارة البيئية، وكذلك داخل بعض المدارس التي لم تطبق نظم الجودة الثاملة المجال البشري: بالنسبة لمجتمع الدراسة فهم المختصون بالعملية التعليمية بالمدارس الحكومية بالمرحلة الابتدائية، المجال الزمني: فترة جمع البيانات وهى فترة أربعة أثشر من شهر أغسطس 2015/إلى ديسمبر • 2015/

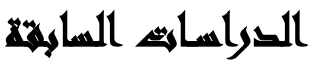

لا شك أن مرحلة الإطلاع على الدراسات والبحوث السابقة حول موضوع الدراسة من

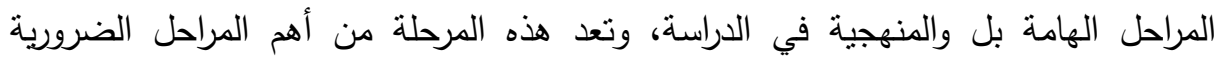

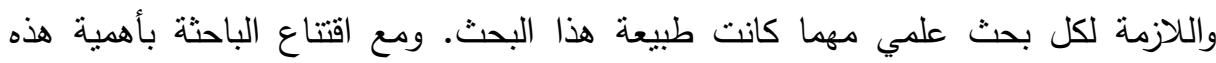
المرحلة فقد حاولت القيام بعمل مسح يكاد يكون عاملا للتراث النظري والدراسات والبحوث السابقة التي أجريت حول موضوع الدراسة الراهنة والقضايا التي تتاولتها هذه الدراسات. دراسة صـلاح حسن سلام I ...ץ،" إدارة الجودة الشاملة كمدخل لتحسين مستوى جودة الخدمة التعليمية في الجامعات المصرية الحكومية"، إطار مقترح رسالة دكتوراه غير منشورة كلية التجارة، جامعة عين شمس: هدفت الدراسة إلى 
البحث على توعية المنظمات بمدخل إدارة الجودة الثاملة في القطاع الحكومي وتحديد منطلبات ومراحل النطبيق، بالإضافة إلى توصيف النقافة للمنظمة السائدة في الجامعات الحكومية المصرية ودراسة أوضاعها الأكاديمية والمالية والإدارية. وقد أسفرت نتائج الدراسة إلى الأتي: عدم ملائمة الثقافة التنظيمية السائدة بالجامعات الحكومية الني تعوق مراحل ومتطلبات تطبيق إدارة الجودة الثاملة، كما أسفرت النتائج بأن هناك فرق بين مستوى جودة الخدمة المقدمة للطلاب بالجامعات الحكومية، ومستوى جودة الخدمة التي تتفق ورغباتهم ونوقعاتهم من حيث الكتاب الجامعي، وإدارة هيئة التدريس

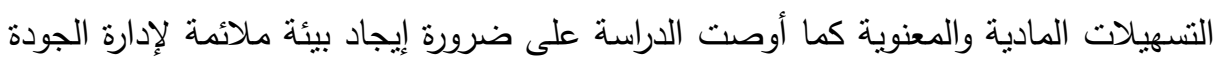

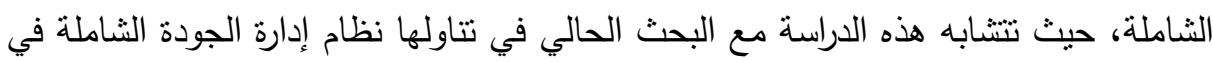
القطاع الحكومي وكذلك في مجال الخدمات التعليمية إلا أن هذه الدراسة تختلف في تطبيقها على بعض الجامعات الحكومية ودراسة الباحث نم تطبيقها على مدارس التعليم الأساسي. دراسة خالد عبد السميع قاسم - بوابة الثانوية العامة المصرية - إصدار • 1 ـ ب بعنوان: الجودة والاعتماد التريوي، من أهم أهداف الدراسة نوضيح مفهوم إدارة الجودة الثاملة :هي مدخل لتحسين منظومة العطلية التعليمية بوجه عام بما تتضدنه من معلم ومتعلم وإدارة مدرسية ومبني مدرسي وبيئة تعليمية ومناخ مدرسي، وما يتطلبه ذلك من دعم

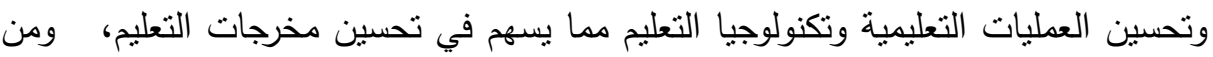
أهم نتائجها :ان نظام توكبد الجودة في التعليم يخدم هدفين اساسيين هما :

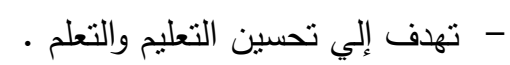

- حساب التكلفة بالنسبة لجودة أنشطة التدريس واستخدام المصادر المتاحة لتحقيق الجودة .

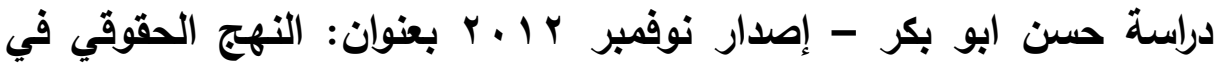
التتمية وتطبيقاته في مجالات البيئة .

من اهم أهداف الدراسة: مراقبة حقوق الطلبة في بيئة خالية من الاضرار داخل المدرسة

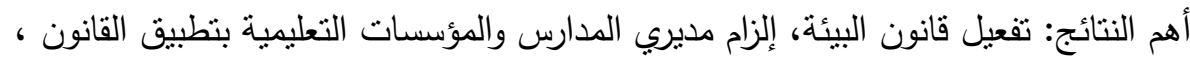
تفعيل الادارة البيئية المدرسية يؤدي إلي التأثنر بإيجابية علي جودة التعليم.

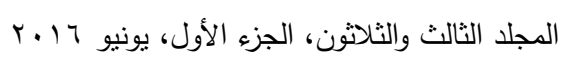




\section{الإسار النزائه}

يتم الوقوف على مختلف المفاهيم والأهداف المتصلة بنظم الإدارة البيئية ومزاياها ثم يليه تقييم لجودة العملية التعليمية

مفهوم الإدارة البيئية: تعني التعديلات المطلوبة في نظم إدارة المنشآت والمنظمات المختلفة، بحيث يكون الاهتمام بالبيئة مجالاً مؤثراً وفعالاً فيها، ويبدو ذلك واضحاً في الهيكل الوظيفي للمنشآت وتحديد المسئوليات والمهام وتتفيذ وإنجاز نظم التخطيط والمراجعة البيئة، ووضع السياسات الملائمة بهدف تحسين أداء المنشأة وخفض آثارها البيئية أو منعها تماماً وقد بدأت الدول في الغرب منذ منتصف الثمانينات ومنذ فترات قريبة في الثرق والبلدان

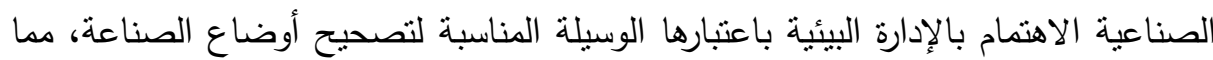
دفع العديد من الحكومات على وضع مقاييس نتريعية للإدارة البيئية وعموماً فأن نظام الإدارة البيئية يتكون من خمسة مراحل تؤدى الى التحسين المستمر :السياسة البيئية، التخطيط، التنفيذ، التقييم، المراجعة

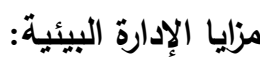
أكثر الأنظمة الإدارية التي ظهرت حتى الآن، من حيث الفاعلية في تحقيق أداء بيئي

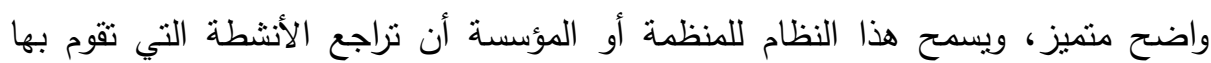

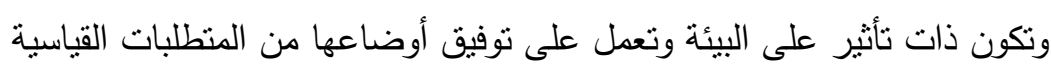

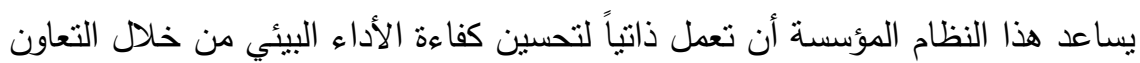

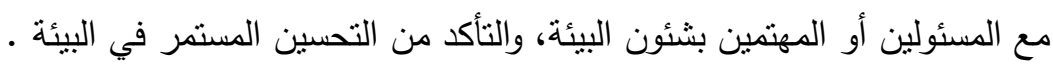
كما يساعد هذا النظام على التأكد من التحسن المستمر واستخدام الأساليب والوسائل

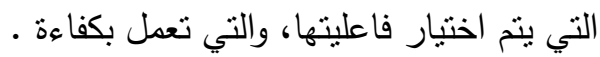

تقييم جودة العملية التعليمية: فإن الجودة في التعليم هي عملية استيفاء النظام التعليمي للمعايير والمستويات المتفق عليها لكفاءة النظام التعليمي وفاعليته بمختلف عناصره) الدخلات ,العمليات ,المخرجات ,البيئة (بما يحقق أعلى مستوى من القيمة والكفاءة والفاعلية 
لكل من أهداف النظام وتوقعات طالبي الخدمة التعليمية ) الطلبة ,المجتمع (وعلى ذلك فهى

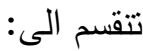
مفهوم العملية التعليمية : تعرف العملية التعليمية بأنها تلك العملية التي تتفاعل فيها ومن خلالها المدخلات المختلفة بنسب ومواصفات معيارية محددة مع المتعلم بشخصيته واتجاهاته

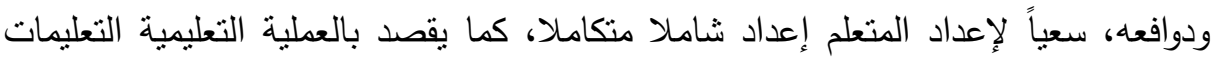
والإجراءات والنشاطات التي تحدد خلل الفصل الدراسي والتي تهدف إلى إكساب المتعلمين معرفة نظرية أو مهارة عملية أو اتجاهات إيجابية عناصر العملية التعليمية: تنكون العملية التعليمية من عدة عناصرة إئهر تعد أساساً لنجاحها وهى كالتالى ( المعلم، المتعلم، المنهج ) المعلم: يعتبر القاسم المشترك الأعظم فى جميع الأنظمة التربوية ويتفق قادة الفكر والمربون

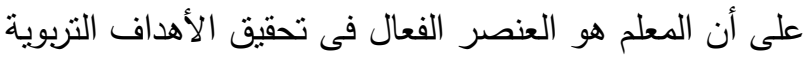

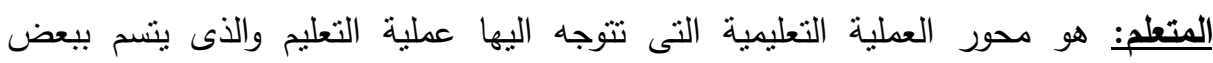

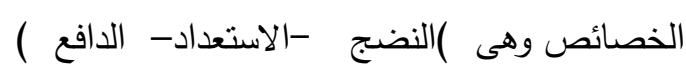

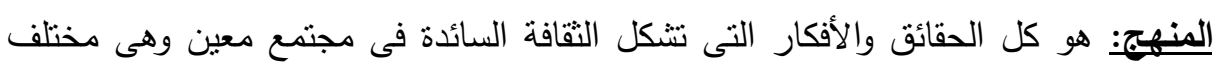

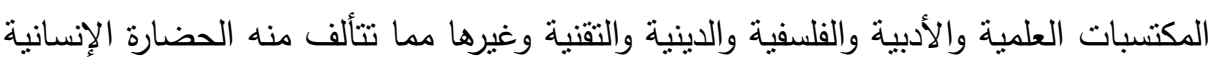

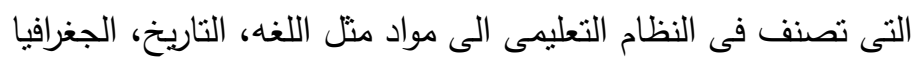

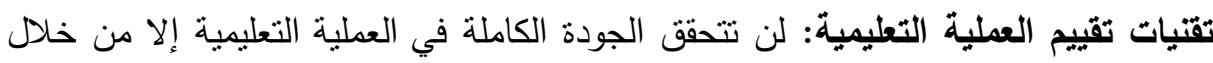

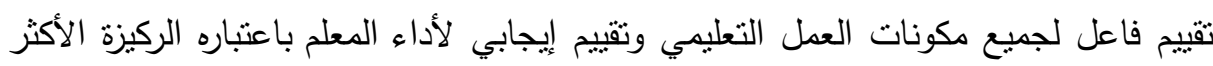

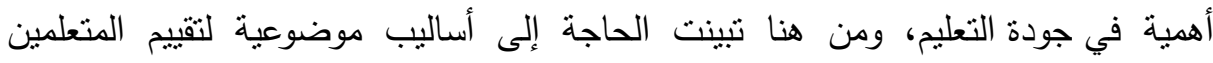
والمعلمين والمؤسسات التعليمية، فالمسأله التربوية تتطلب من النظام التعليمي إيجاد طريقة، تؤسس التقة في مقاييس تقييم مخرجات المؤسسات التعليمية المتمنلة في نواتج التعلم المستهذفة. 
وتأسيساً على ذلك سوف نتناول فيما يلي جودة التعليم ومعاييرها السائدة الجودة الشاملة في التعليم: هي القيمة المضافة التي تعني مدى قدرة العملية التعليمية على لـى تطوير معارف ومهارات وقدرات المتعلم معايير مؤسسات التعليم قبل الجامعى: وتبدأ بقيام المؤسسة بدراسة ذاتية لأدائها بمختلف ولفارت

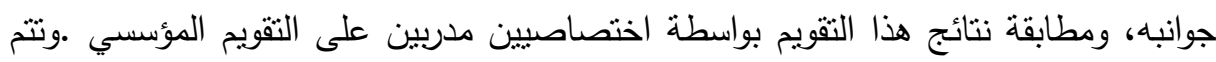
هذه العملية في ضوء معايير ضمان الجودة والاعتماد الصادرة من الهيئة القويمة لضمان

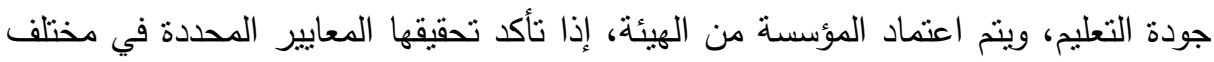

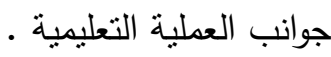
معايير نظم التقويم داخل المؤسسات التعليمية: وفي هذا الثأن قامت الهيئة القويمة لضمان جودة التعليم والاعتماد بوضع معايير التقويم المتمثلة في:

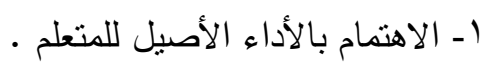

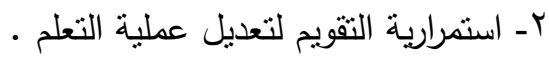

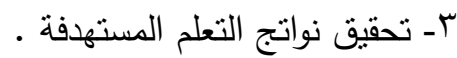

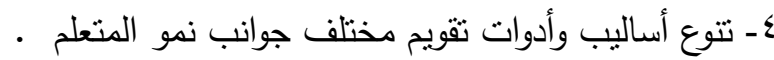

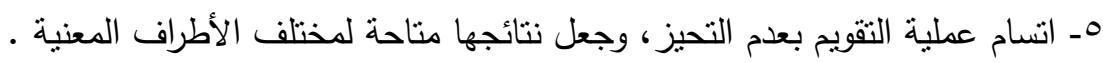
وترجع أهمية دور التقويم التريوى فى إصلاح التعليم الى عدة عناصر من أهمها:

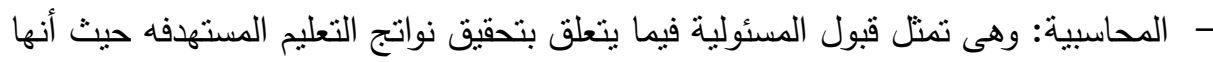
تؤكد حق جميع الأطراف المعنية فى معرفة ما يجرى فى المؤسسات التعليمبة.

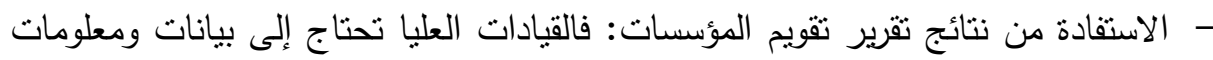
تتعلق بمسنوى أداء المؤسسة التعليمية في تحقيق الأهداف، أو المستويات المرجوة لمناقتشة وتعزيز جوانب القوة، وكيفية التغلب على جوانب الضعف وتعرف أسبابها التي ربما ترجع إلى انخفاض مستوى المعلمين، أو نقص الإمكانات البشرية والمادية، من أجل تطوير 


\section{الإطال المزهيهي اللهحهي}

حيث استخدم الباحثون في جمع بيانات الدراسة الميدانية أسلوب قائمة الاستقصاء، وفي ضوء ذلك يعرض الباحثون مراحل إعداد قائمة الاستقصاء، ثم تقدم وصفاً لمجتمع وعينة لأنة الدراسة، والإجراءات التي اتبعت في توزيع قائمة الاستقصاء وطريقة جمع البيانات، والأساليب الإحصائية المستخدمة فى التحليل والوصول إلى نتائج البحث جلول( (1): الخصائص الديموجرافية لعينة البحث

\begin{tabular}{|c|c|c|c|}
\hline 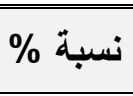 & عدد & 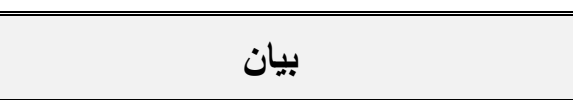 & الإيموجرائصية \\
\hline Tr, & 159 & مدرس & \multirow{7}{*}{ الوظيفة } \\
\hline$r M, Y$ & $\varepsilon \Lambda$ & مدرس أول & \\
\hline $7, \wedge$ & 14 & وكيل & \\
\hline$r, \varepsilon$ & 0 & مدير & \\
\hline $1, \varepsilon$ & $r$ & موجه & \\
\hline$r, q$ & $\Lambda$ & أخصائيون & \\
\hline $1 \ldots$ & $r \cdot T$ & الإجمالي & \\
\hline $1 \wedge, \cdot \varepsilon$ & $r \wedge$ & فوق المتوسط & \multirow{4}{*}{ الدرجة العلمية } \\
\hline$V V, r$ & 109 & جامعي & \\
\hline$\varepsilon, \varepsilon$ & 9 & أعلي من جامعي & \\
\hline $1 \ldots$ & $r \cdot T$ & 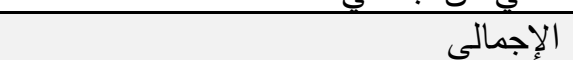 & \\
\hline 0,1 & 11 & أقل من 5سنوات & \multirow{6}{*}{ الخبرة في مجال } \\
\hline$\pi r, \cdot 1$ & $1 \pi$. & من • سنوات إلى أقل من • أسنوات & \\
\hline$\Gamma_{1, \cdot T}$ & 70 & • . سنوات فأكثز & \\
\hline $1 \ldots$ & $r \cdot T$ & الإجمالي & \\
\hline \multicolumn{2}{|c|}{ 9,10 سنة } & المتوسط الحسابي لسنوات الخبرة & \\
\hline \multicolumn{2}{|c|}{ V Y, Y } & الانحراف المعياري & \\
\hline
\end{tabular}

قام الباحثون بترميز أسئلة قائمة الاستقصاء حيث تم تعريف متغيرات الدراسة عن طريق

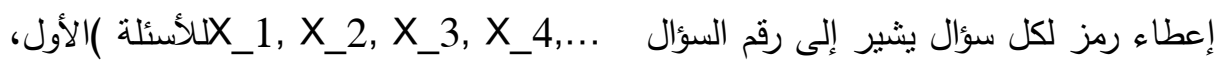

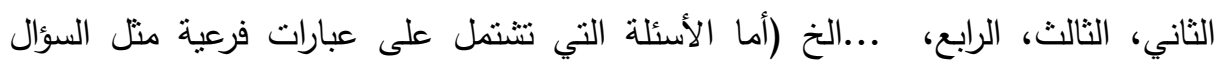

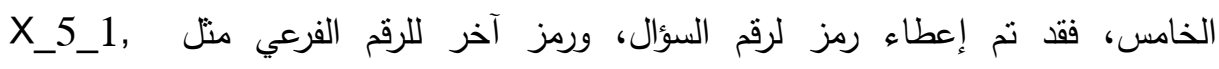


اللسؤال الخامس العبارة الأولى، والسؤال الخامس العبارة الثانية ....الخ، وقد تم

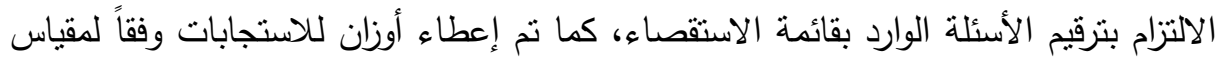

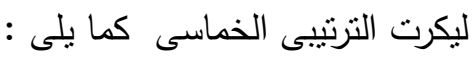

\begin{tabular}{|c|c|c|c|c|}
\hline غليى الاطلاقق & غير موافق & محايل & موافق & موالأقيلق على \\
\hline 1 & $r$ & $\Gamma$ & $\varepsilon$ & 0 \\
\hline
\end{tabular}

جدول(ץ): مقاييس الاعتمادية كما جاءت من تحليل قائمة الاستقصاء وفيما يلي تطبيق هذه الأسالبب الإحصائية على متغيرات الدراسة: هائه التحليل الإحصائي وإختبار الفروض:

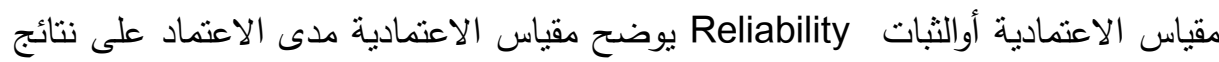

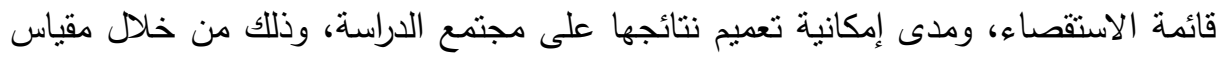

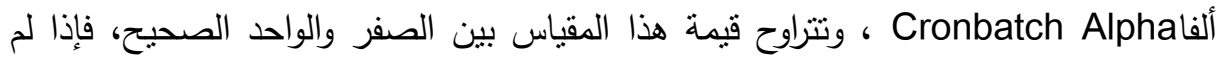
يكن هناك ثبات فى البيانات فإن قيمة هذا المعامل تساوى صفراً، وعلى العكس إذا كان هنآك

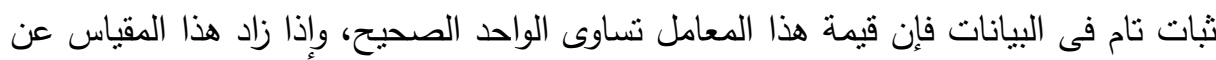

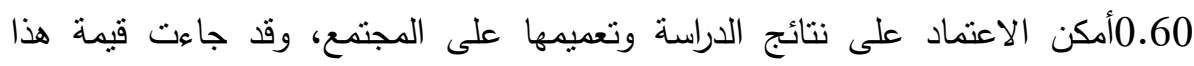

\begin{tabular}{|c|c|c|c|c|}
\hline 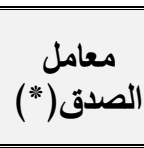 & معامل & 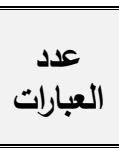 & توصيف السؤال & 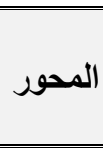 \\
\hline$\% q r, \vee$ & $\% \wedge \neg, 1$ & ru & تطبيق الإدارة البيئية في المثلدارس التي تطبيق الجودة & 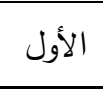 \\
\hline$\% 9 r, 7$ & $\% \wedge \bullet, \vee$ & $\leqslant 9$ & 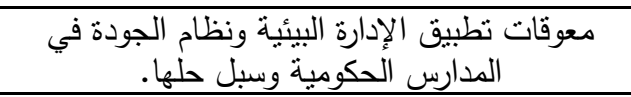 & 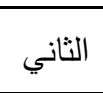 \\
\hline$\% \vee 9, \wedge$ & \%тr, & $\varepsilon$ & تأثير نطبيق نظم الإدارة البيئية على جودة التعليم & 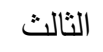 \\
\hline
\end{tabular}

(") تم حساب معامل الصدق عن طريق جذر معامل الثبات

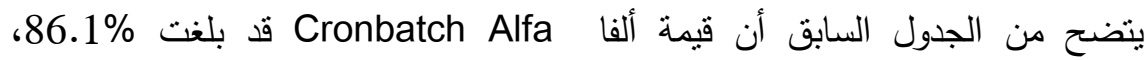
85.7\%، 63.7\% على محاور قائمة الاستقصاء على التوالي، مما يدل علي أنها تتمتع 
بالصلاحية Reliability الأمر الذى يمكن معه الاعتماد على النتائج وتعميم هذه النتائج

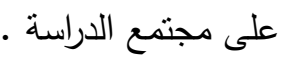
الإحصاء الوصفي ونتائج اختبار T: بإجراء اختبار T عن القيمة المحايدة، جاءت النتائج كما يلي: جدول(ץ): الإحصاء الوصفي ونتائج اختبار T لتطبيق الإدارة البيئية في الددارس

\begin{tabular}{|c|c|c|c|c|c|c|}
\hline الدالة & مسنتوية & $\begin{array}{c}\mathrm{T} \\
\text { test }\end{array}$ & المعياري & مرجح & وصف المتغير & المتغير \\
\hline غالةيز & $\cdot, 7 Y 1$ & $\cdot, \leqslant 97$ & $1, \varepsilon 1$ & $r, 00$ & 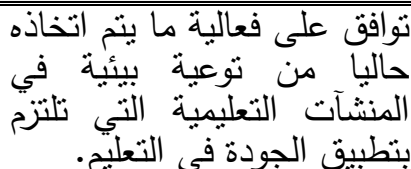 & X_1 \\
\hline موجبة & ס T., & $r,|Y|$ & $1, .0$ & ד, & 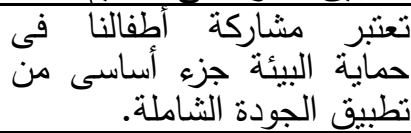 & X_2 \\
\hline دالةيز & $\cdot, \cdot \vee \wedge$ & $1, \vee \vee 1$ & $1, \cdot 7$ & r,T & 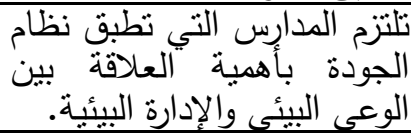 & X_3 \\
\hline دوجبة & $\cdot, \cdot, \cdot$ & $Y, T \cdot T$ & $\cdot, 9 \leq$ & $\Gamma, T V$ & 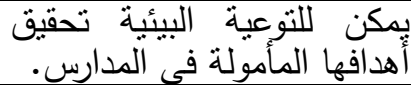 & X_4 \\
\hline
\end{tabular}

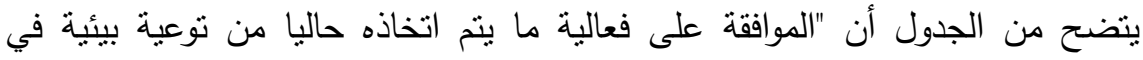

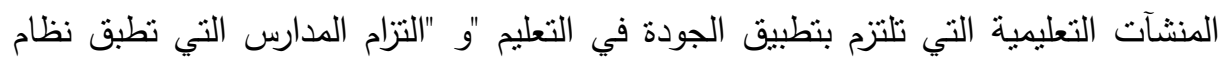
الجودة بأهمية العلاقة بين الوعي البيئي والإدارة البيئية "جاء محايداً، أي أنه لم يصل إلى لئى حيز

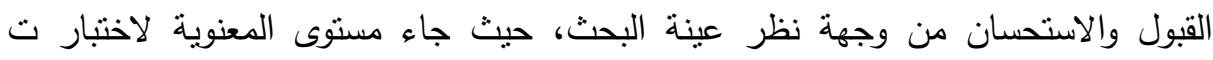

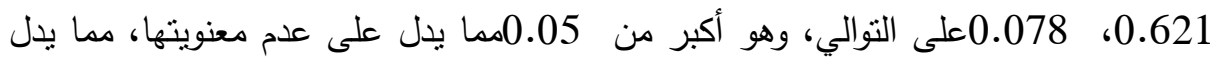

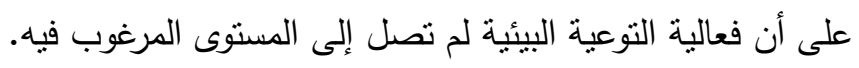

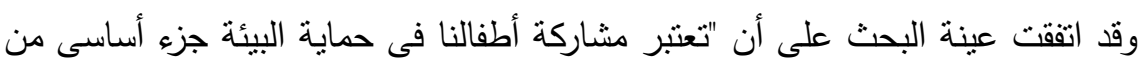

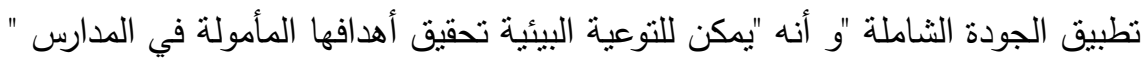




\section{أهم نتمائي الصراسلة}

أولاً:النتائج النظرى: توضيح دور نظم الإدارة البيئية داخل المدارس:

لقد أوضحت نتائج الدراسة أن تطبيق نظم الإدارة داخل المدرسة في ذلك يعطي للمعلمين

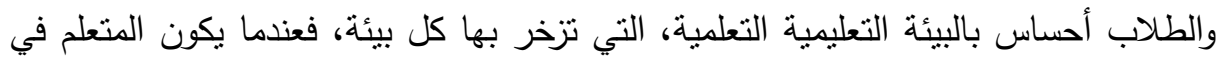

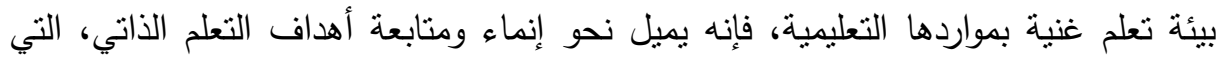

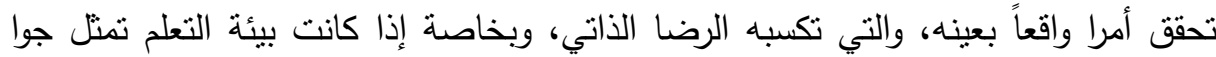

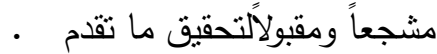
قياس إنطباع المدرسة عن نظم الإدارة البيئية حيث أوضحت الدراسة أن نظام الإدارة البيئية

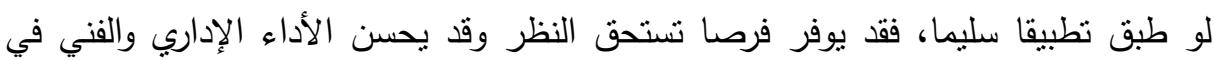

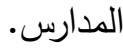

توضيح الجدوى من تفعيل نظم الإدارة البيئية حيث أوضحت الدراسة أن الإدارة البيئية تتمي قدرة الطالب على إدراك أهمية البيئة في التتمية الاقتصادية والثقافية، ومن ثم ترسخ الثُعور بالمسئولية تجاه البيئة والمحافظة على صحة البيئة الطبيعية والاجتماعية والثقافية ثانياً: النتائج العملى: باهن: باهن تم قبول الفرض الأول: " يوجد علاقة ارتباط موجبة بين نطبيق نظم الإدارة البيئية في الدداس

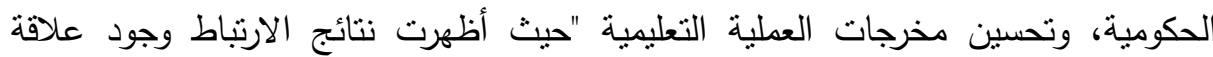
ارتباط موجبة ذات دلالة معنوية بين المتغير التابع "جودة التعليم "وكلٍ من التغيرات "أهداف

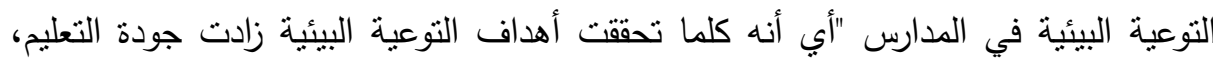

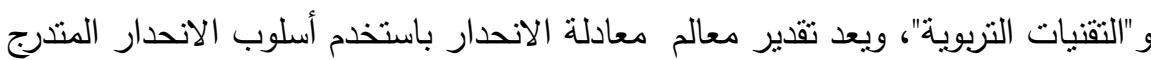
تم قبول الفرض الثاني: "يؤدي وجود معوقات لتطبيق نظم الإدارة البيئية إلى ضعف مخدئ مخرجات

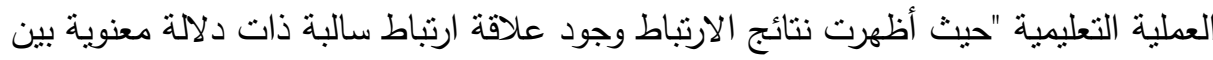
المتغير التابع "جودة التعليم "وكلٍ من المعوقات التي تعرقل التحول من المدرسة التقليدية إلى الى لهديه

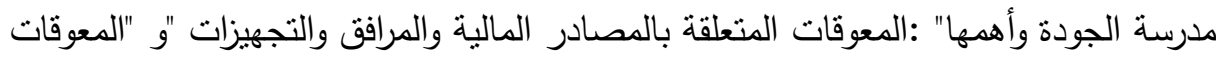
المتعلقة بالعلاقة بين المدرسة ووزارة التربية والتعليم "و "معوقات ذات صلة بالعملية التعليمية " 390

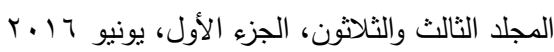


جمال سعد خطاب وآخرون

وكذللك "القصور في الأجهزة القائمة على تتفيذ التشريعات لمكافحة التلوث "و "عدم وعى

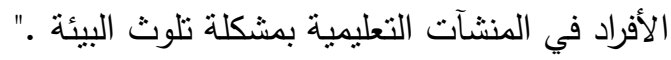

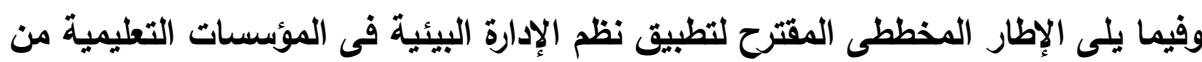
إعداد الباحثة بناءًا لما تم التوصل إليه من نتائج:

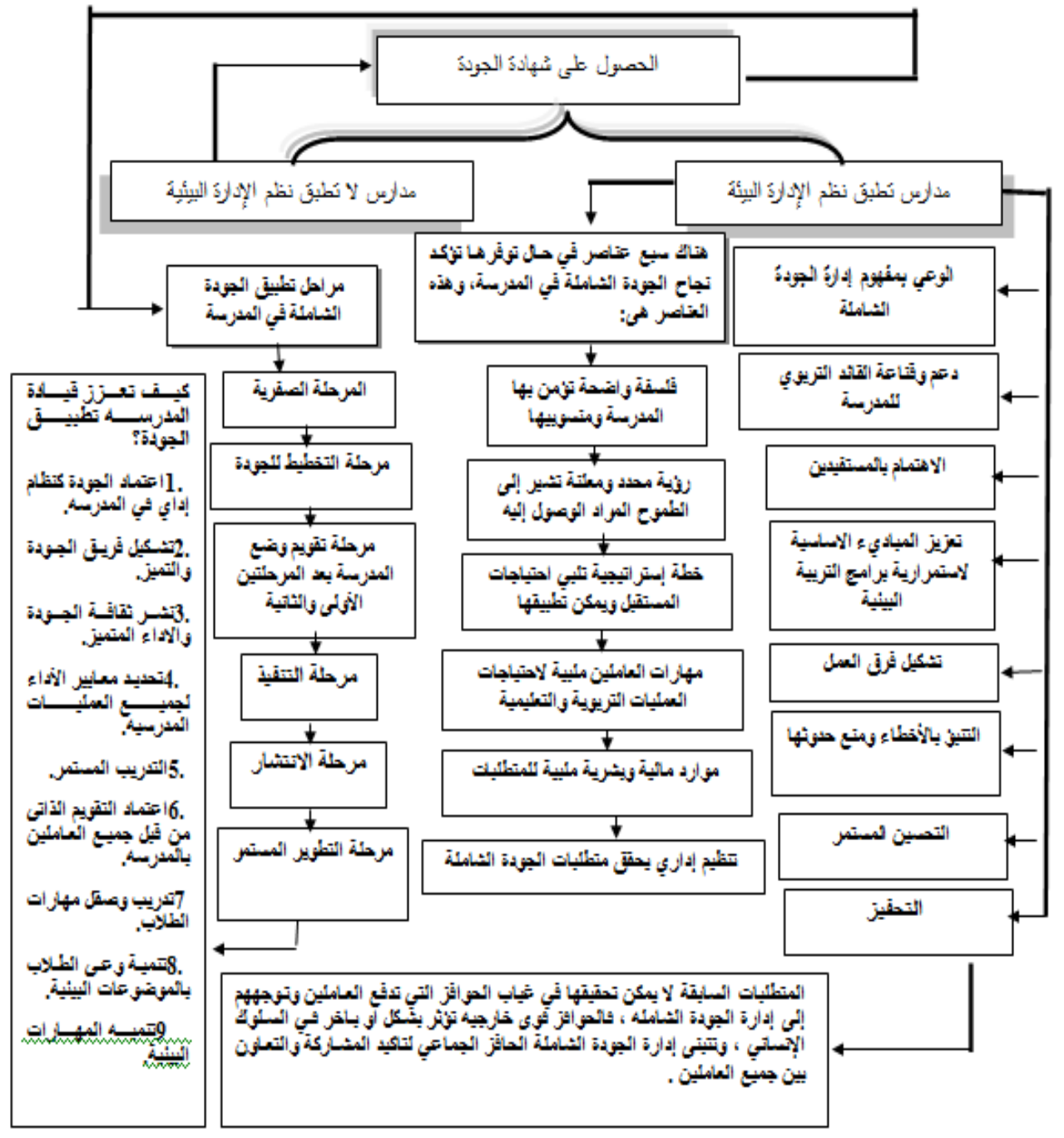

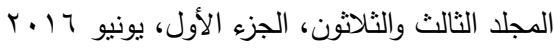




\section{تموسيايت التراسة}

ا. إدراج التربية البيئية كأحد أركان المنهج التربوي وتضمين المناهج الدراسية إمداد الأطفال بالمعلومات والحقائق والمفاهيم البيئية في المجالات والتخصصات المختلفة، وتتمية التئية مهاراتهم التي تساعدهم على صيانة بيئتهم وتتمية مواردها، مع إكساب التلاميذ القيم والاتجاهات الإيجابية نحو حماية البيئة وتتميتها وذللك لتوضيح دور نظم الائه الادارة البيئية داخل المدارس. r. التعاون بين وزارتي التربية والتعليم والبيئة من أجل التعريف بكل ما هو جديد في مجال

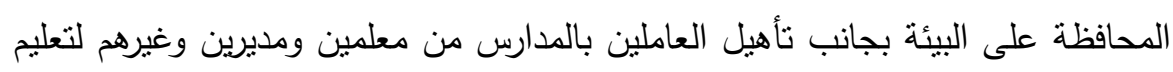

$$
\text { ونشر ثقافة التربية البيئية والوعي البيئي. }
$$

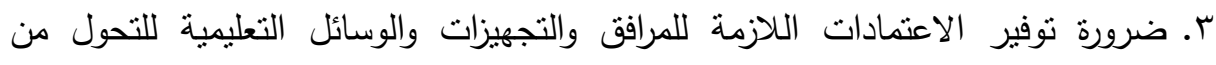
المدارس التقليدية إلى مدارس الجودة، وفق خطة مدروسة تتلاءم مع الإمكانيات المتاحة، ووفقاً لجدول زمني يتم متابعة تتفيذه. ع. استخدام الأنشطة التعليمية وطرائق التدريس التي أثنتت فعاليتها في كسب ونتب وتتمبة المفاهيم والاتجاهات البيئية لدى طالبات شعبة الطفولة منل الدراسات الحقلية، واستخدام أساليب البحث الميداني في مجال التربية البيئية كالملاحظة والمقابلة . ه. تتويع أساليب التقويم لكي يشمل الجانب النظري من المنهاج والجوانب العملية أيضا منها: المسابقات، واستبانات قياس المعرفة والاتجاه، وبطاقات السلوكات البيئية. 


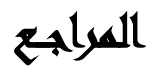

السيد عبد النور عبد الباري( . . ؟؟): "تلوث البيئة ـالأرض والنبات"، دار النشر للجامعات

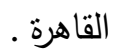

سيد إبراهيم الجيار ( . . †): "دراسات في تاريخ الفكر التربوي"، دار هناء للنشر، بيروت، .

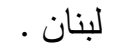

رعد حسن الصرن الصرن( ( . ؟): "نظم الإدارة البيئية والأيزو "14000، دار الرضا للنشر،

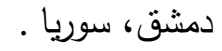

سهيلة محسن كاظم القتلاوي(ب . . ץ): "المدخل الى التدريس"، دار الثروق للطباعة، رام الله، 2. المنارة، طن الفناوي

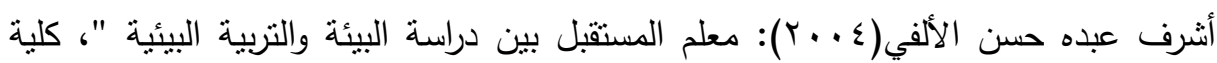

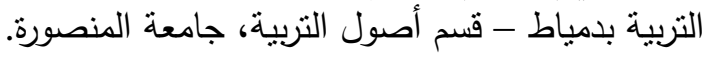

نجلاء فتحي الدسوقي( ( . ب): "سياسات نرشيد الطاقة في جمهورية مصر العربية"، رسالة ماجستير، غير منشورة، قسم الإدارة العامة، كلية الاقتصاد والعلوم العيدة السياسية، جامعة القاهرة، مايو . مانير

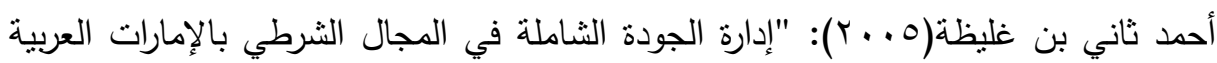

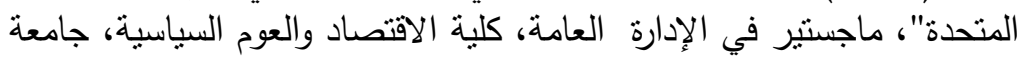

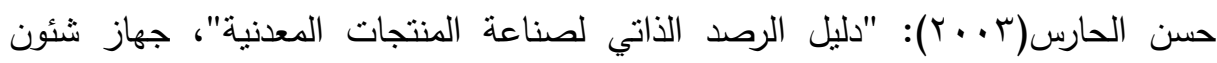

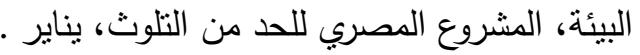

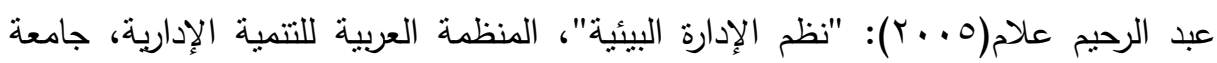
الدول العربية.

Albert Edmonton:(1999): "Educational quality indicators" Annotated Bibliography, cdition Canada.

Daniel Minoli :(2007): Distance Learning, Technoloby and application , Boston, Artech House.

Egyptian Pollution Abatement Project "Summary of Pipeline Projects" May 2008.

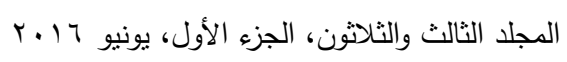


Evans, J, (1997): "Applied production and operation Management , 4th , ed .West publishing Co.

GETF, The USEPA Environmental Management System Pilot Program for Local Government Entities, January 2010.

Gilluespic, John and Spirt, Dinal, Administering, The School Library Media Center, New Yourk : Bowker company, 1993.

Haksen \& others,(2011): "Service management and operations", 2ns edition, prentice-hall upper saddle river, new jersey.

Principal etal., (1999): EMS : An Implementation Guide for SMOS , NSF , USA .

\title{
APROPOSED FRAMEWORK FOR APPLYING ENVIRONMENTAL MANAGEMENT SYSTEMS IN GOVERNMENTAL SCHOOLS AND ITS IMPACT ON QUALITY OF EDUCATION
}

Khatab, G. S. ${ }^{(1)}$; Mohamed, Reham, R. $^{(2)}$ and Saleh, Sahar, N. M. 1) Faculty of Commerce, Ain Shams University 2) Institute of Environmental Studies and Research, Ain Shams University

\begin{abstract}
In fact, School environmental management systems seek making human beings enjoy having skills and attitudes due to quality of concepts included within educational curricula. Total quality is an administrative strategic process that is based on group of values that derive their motional energy from information in terms of which we are able to employ workers' talents in educational institutions creatively.
\end{abstract}

$$
\text { المجلد الثالث والثلاثثن، الجزء الأول، يونيو } 17 \text { r. }
$$


جمال سعد خطاب وآخرون

The study is not limited to specific method, but its follows a two-fold method, first, the inductive approach, in terms of the theoretical framework of the research, problem and hypotheses. Second, the analytical approach as the study counts on the qualitative-analytical method which is divided into secondary resources approach that depend on using references and available in libraries, books, studies, and essays; the primary approach as an applied study on governmental schools. Researcher use as study tools: monitor card - an observation form for environment and school environmental behavior - an investigation form containing several questions, to be fulfilled through personal interviews. The form's purpose is obtaining information, facts, and data concerning the value of applying environmental management systems in governmental schools that adopt the total quality concept. The study uses several statistical treatments and tools to be used for the field study and applied upon governmental primary schools as the most important age stage for students. The research has extracted multiple results and suggestions concerning activities at schools to administer the environmental management systems and focus on environmental education in terms of rules of total quality. Results indicate the necessity for school environment's coping with new educational needs and the development of student- teacher relationship. Consequently, organizational culture of total quality could be prevailed and dominant and the environmental values and philosophy will be also diffused in primary stage as a basic natural basis for improving quality of education process. 